\title{
Trends in condom use among female sex workers in Andhra Pradesh, India: the impact of a community mobilisation intervention
}

\author{
Jennifer Toller Erausquin, ${ }^{1,2}$ Monica Biradavolu, ${ }^{3,4}$ Elizabeth Reed, ${ }^{5}$ \\ Rebekah Burroway, ${ }^{6}$ Kim M Blankenship ${ }^{3,4}$
}

${ }^{1}$ Duke Global Health Institute, Duke University, Durham, North Carolina, USA

${ }^{2}$ North Carolina Division of Public Health, Raleigh, North Carolina, USA

${ }^{3}$ Centers on Health, Risk and Society, American University, Washington, District of Columbia, USA

${ }^{4}$ Department of Sociology, American University, Washington, District of Columbia, USA ${ }^{5}$ Department of Prevention and Community Health, George Washington University, Washington, DC, USA

${ }^{6}$ State University of New York at Stony Brook, Stony Brook, New York, USA

\section{Correspondence to} Dr Jennifer Toller Erausquin, North Carolina Division of Public Health, 1928 Mail Service Center, Raleigh, NC 27699-1928, USA jt.erausquin@gmail.com

Accepted 28 February 2012 Published Online First 11 April 2012

\section{ABSTRACT}

Background Community mobilisation interventions for HIV prevention among female sex workers (FSWs) aim to organise FSWs for collective action and challenge the structures of power that underlie HIV risk. Assessing intervention impact is challenging because the importance of direct individual exposure to intervention components may decrease over time as change occurs at social-normative, policy and other structural levels. In this paper, the authors examine changes over time in consistent condom use among FSWs in Rajahmundry, Andhra Pradesh, the location of a long-standing community mobilisation intervention.

Methods The authors analyse cross-sectional data collected among FSWs at three time points ( $n=2276$ ) using respondent-driven sampling. Multiple logistic regression was used to assess the association of programme exposure with consistent condom use and whether this association varied over time.

Results The proportion of FSWs having no exposure or only receptive exposure to the intervention decreased over time, while active utilisation increased from 19.4\% in 2006 to $48.5 \%$ in 2009-2010. Consistent condom use with clients also increased from $56.3 \%$ in 2006 to $75.3 \%$ in 2009-2010. Multivariate analysis showed that age, age at start of sex work, venue, living conditions and programme exposure were significantly associated with condom use. The positive association between programme exposure and consistent condom use did not vary significantly over time.

Conclusions Findings indicate improvements in HIV risk reduction behaviour among FSWs and suggest that the intervention has substantial reach in the FSW population. The intervention's strategies may be contributing to population-level HIV risk reduction among FSWs.

\section{INTRODUCTION}

In India and around the world, female sex workers (FSWs) are a group at particular risk for HIV infection. In India, although general population prevalence is below $1 \%(0.3 \%$ as of 2009$)$, an estimated $5 \%$ of FSWs are living with HIV. ${ }^{1}$ Rates are even higher in southern India, where HIV prevalence among FSWs is estimated to be $14.5 \%$, ranging from $2 \%-38 \%$ across districts. ${ }^{2}$ The southern Indian state of Andhra Pradesh is among those experiencing the highest impact of HIV/ AIDS. ${ }^{3} 4$ HIV prevalence among FSWs in Andhra Pradesh has been estimated to be as high as $16 \%,{ }^{5}$ and as a result, a number of HIV prevention programmes targeting FSWs are being implemented in the state. ${ }^{4}$ In other south Indian settings, current HIV prevention interventions have been associated with reduced HIV risk behaviours and declining HIV risk among FSWs. ${ }^{6}$

Understanding and addressing HIV risk among FSWs requires attention to both individual risk behaviours and social-structural factors that shape the contexts of risk. Stigmatisation, disempowerment and socioeconomic marginalisation of FSWs create and reinforce their risk for $\mathrm{HIV}^{8}$ These dynamics have led to an increased recognition of stigma reduction as a key component in HIV prevention and treatment, ${ }^{9-11}$ and the significance of community-level and structural factors in HIV/AIDS transmission and mitigation. ${ }^{12} 13$

A broad literature exists on the impact of HIV risk reduction interventions for FSWs ${ }^{14}$ and several successful interventions have incorporated community-level and structural change components. In India, interventions for FSWs have been linked to increased knowledge of HIV risks ${ }^{15}$; consistent condom use with clients ${ }^{16-18}$; condom use with occasional clients, regular clients and non-client partners ${ }^{6} 7$; and reduced prevalence of sexually transmitted infections (STIs). ${ }^{6} 7$

A common thread among these interventions is their structural approach. Broadly speaking, structural interventions aim to alter the risk-creating environments of the social and physical environments. ${ }^{13} 1920$ Structural interventions promote HIV prevention by addressing these environments, increasing the behavioural choices available to individuals and reducing barriers that prevent behavioural change. Community mobilisation interventions (CMIs) are a type of structural intervention that emphasises changes to power relations between groups. ${ }^{16}$ CMIs often involve multiple activities, such as consciousness raising among marginalised groups, engaging in advocacy with stakeholders who hold power and contesting barriers to preventive behaviours. ${ }^{13}$ Many CMIs also incorporate individual behaviour change strategies, such as STI/HIV education or testing. For example, the Sonagachi project in Kolkata, India, provides treatment for STIs and promotes normative change in health behaviours and empowerment through the mobilisation and active participation of FSWs. ${ }^{21}$ Sonagachi has been widely touted as a successful model for community-led structural interventions. ${ }^{17} 2122$

Although structural interventions generally and CMIs in particular represent a promising approach for supporting sustainable behaviour change, there 
are a number of challenges associated with evaluating them. ${ }^{13}$ Normative and other social-environmental changes may or may not require direct exposure to or participation in intervention activities. ${ }^{20}$

In this paper, we explicitly examine the role of receptive exposure (ie, awareness, receiving a pamphlet or being visited by intervention staff) and active utilisation (ie, visiting the intervention centre or using medical services) of an FSW CMI in the Rajahmundry area of Andhra Pradesh. The CMI is part of the Bill and Melinda Gates Foundation's Avahan India AIDS initiative (Avahan) and was begun in 2004. The CMI had initially been implemented by the local branch of an international nongovernmental organisation headquartered in the USA but is currently in the process of transitioning to full Indian ownership and oversight. The intervention organises FSWs to build collective power and channels this collective power to address structural determinants of HIV risk, including stigma, policing policies and practices, condom availability, and access to STI testing and treatment and to loans to address economic vulnerability. ${ }^{23}$ It encourages FSWs to form community-based organisations comprising and run by FSWs. The intervention also uses 'social change agents', local FSWs who serve as peer health educators and community organisers. FSWs mobilised by the intervention hold public rallies and media events, meet with public officials and community groups to promote awareness of FSW issues, assist with intervention-run STI clinics and respond to FSW complaints of police mistreatment. The strategies of this CMI thus include both individual behaviour change (eg, peer health education) and efforts to impact social norms and public policy (eg, working with police to improve police treatment of FSWs). Data collected as part of this CMI provide a unique opportunity to examine whether direct exposure to intervention components remains important over time for improvements in FSWs' HIV risk reduction behaviour.

The analyses in this paper focus on three research questions: First, what is the level of consistent condom use with clients over time among FSWs in Rajahmundry? Second, what is the relationship between exposure to the CMI and consistent condom use with clients? Finally, does the association between intervention exposure and consistent condom use vary over time?

\section{METHODS}

\section{Data source}

Data for this analysis were collected as part of Project Parivartan, a larger study of the implementation and impact of a CMI for HIV prevention in the Rajahmundry area of East Godavari district of Andhra Pradesh. We analysed cross-sectional survey data collected at three time points: April to June $2006(n=812)$, March to May 2007 ( $n=673$ ) and November 2009 to February $2010(n=850)$. Participants were eligible for the study if they were female, at least 18 years old and reported exchanging sex for money at least once in the 12 months prior to the survey.

In each round of data collection, respondent-driven sampling (RDS), a modified chain referral method, was used to recruit participants. The process began with five initial participants ('seeds'), who were selected to represent diverse groups of FSWs. Following their interviews, they were asked to distribute up to three coupons to other eligible FSWs in their social networks. Participants who met the eligibility criteria and came into the study with a coupon were, in turn, given three coupons to recruit members of their social networks; this process continued until the predetermined sample size was achieved. (The survey was anonymous and therefore we were unable to determine which respondents participated in more than one round of data collection.) As each survey round had a high number of recruitment waves (ie, each seed recruited women who recruited more women and so on), we are confident that the RDS method resulted in adequate coverage of the FSW population in Rajahmundry area. ${ }^{24}$

Interviews lasted approximately $90 \mathrm{~min}$ and were conducted in the local language (Telugu) by trained interviewers after participants provided informed consent. Participants received modest monetary incentives for both completion of the interview and successfully recruiting other FSWs into the study. The research protocol was approved by ethical review boards at American University, Duke University, Yale University and YRG-Care, Chennai, a research institution in India.

Of the 2335 respondents, 59 were missing data on one or more study variables and were excluded from all analyses, resulting in an analytic sample of 2276 FSWs. The proportion of missing data did not vary significantly by survey round.

\section{Study variables}

\section{Consistent condom use with clients}

Consistent condom use with clients is the outcome measuring HIV-related risk behaviour. The respondent was considered to use condoms consistently with clients if, in the past 7 days, she always used condoms with all types of clients she had (ie, regular and/or occasional clients) and used condoms the last time she had sex with a client.

\section{Consistent condom use with non-clients}

For descriptive analyses only, we also examined consistent condom use with husbands, boyfriends and lovers, for respondents who indicated having this type of sexual partner. The respondent was considered to use condoms consistently if, in the past 7 days, she always used condoms with her husband, boyfriend or lover.

\section{Demographic and sex work characteristics}

Age was measured continuously. Age at start of sex work was measured as categories (began sex work at younger than 18 years, $18-29$ years, 30-39 years and 40 years or older). We assessed economic vulnerability with two variables: living conditions, a continuous variable based on whether a respondent indicated she currently lived in a place with electricity, running water, toilet or telephone (values range from $0-4$ ), and debt, a dichotomous variable based on whether the respondent reported having current debt (yes/no). Literacy was indicated by respondent reports of being able to read (yes/no). Venue was measured by asking respondents the venue where they engaged in sex work most recently (brothel, street, lodge or hotel, home, highway, agriculture or other settings or multiple venues). Number of clients in the past 7 days was measured continuously and collapsed into a dichotomous variable for high frequency of sex trades (10 or fewer clients/11 or more clients) based on preliminary sensitivity analyses that suggested this was a natural split for predictive effects.

\section{Programme exposure}

We assessed multiple dimensions of exposure to the CMI. We asked respondents if they had ever heard of the intervention; we also asked them if, in the last 6 months, they had received a pamphlet about the intervention, had been visited by intervention staff, had gone to an intervention-sponsored drop-in centre and/or sought medical services at an intervention clinic. We defined receptive exposure only as having ever heard about the intervention, received a pamphlet or been visited by intervention 
staff, and active utilisation as having visited a drop-in centre or having been to the intervention for medical services. Respondents who had not heard of the intervention were coded as having no exposure.

\section{Data analysis}

We first examined descriptive statistics to compare the demographic and sex work characteristics of FSWs in each of the three survey rounds. Next, we conducted bivariate analyses to assess possible factors associated with consistent condom use with clients. Finally, we used multiple logistic regression to assess the association of programme exposure with consistent condom use with clients. Our initial regression model assessed the programme exposure main effect; subsequent models added (1) sample characteristics identified in bivariate analyses to be associated with condom use at the $p<0.10$ level and (2) interactions with survey round to examine whether the association between programme exposure and condom use varied over time. All statistical analyses were conducted using Stata 10.1/SE, ${ }^{25}$ and no RDS weights were employed.

\section{RESULTS}

The repeated cross-sectional data showed some stability and some change in both demographic and behavioural characteris- tics over time (table 1). On average, FSWs were 32 years old, one-quarter were literate and they reported having 10 clients in the 7 days prior to the survey. The most common sex work venues were homes, agriculture settings, highways and multiple venues. Across the three rounds of data collection, the proportion of respondents working on highways decreased, while the proportion working in multiple venues increased. The proportion of FSWs having no exposure or only receptive exposure to the intervention decreased over time, while active utilisation of the intervention increased from $19.4 \%$ in 2006 to $48.5 \%$ in 2009-2010. Consistent condom use with clients also increased from $56.3 \%$ in 2006 to $75.3 \%$ in $2009-2010$.

Each of the demographic and sex work characteristics included in table 1 was significantly associated with consistent condom use in bivariate analyses $(p<0.10)$, with the exception of current debt $(p=0.98)$ (results not shown). Both age and age at start of sex work were negatively associated with consistent condom use, with older FSW and FSW who started at older ages having lower proportions of consistent condom use with clients. Notably, consistent condom use was dramatically lower among women who entered sex work at age 40 or older (41.7\% always using a condom with clients in the past 7 days compared with $58 \%-77 \%$ among women who entered sex work at younger ages). Literacy was positively associated with condom use:

Table 1 Sample characteristics by survey round

\begin{tabular}{|c|c|c|c|c|c|}
\hline & Round 1, 2006, n=794 & $\begin{array}{l}\text { Round 2, 2007, } \\
\mathrm{n}=669\end{array}$ & $\begin{array}{l}\text { Round 3, 2009-2010, } \\
\mathrm{n}=813\end{array}$ & Total, $\mathbf{n}=\mathbf{2 2 7 6}$ & p Value* \\
\hline Mean age in years (SD) & $32.1(8.24)$ & $32.5(7.84)$ & $32.7(8.4)$ & $32.5(8.21)$ & 0.159 \\
\hline Mean no. of clients, past 7 days (SD) & $10.5(12.23)$ & $10.6(13.63)$ & $9.51(11.28)$ & $10.2(12.43)$ & 0.159 \\
\hline \multirow[t]{2}{*}{ Living conditions index† (range: $0-4$ ) (SD) } & $1.27(1.11)$ & $1.70(1.23)$ & $2.12(1.25)$ & $1.71(1.25)$ & 0.000 \\
\hline & Percentage (n) & Percentage (n) & Percentage (n) & Percentage (n) & \\
\hline Age at start of sex work & & & & & 0.000 \\
\hline$<18$ years & $15.1(120)$ & $17.2(115)$ & $20.9(170)$ & $17.8(405)$ & \\
\hline $18-29$ & $62.3(495)$ & $63.1(422)$ & $65.8(535)$ & $63.8(1452)$ & \\
\hline $30-39$ & $19.7(156)$ & $17.0(114)$ & $12.4(101)$ & $16.3(371)$ & \\
\hline 40 years or older & $2.9(23)$ & $2.7(18)$ & $0.9(7)$ & $2.1(48)$ & \\
\hline Literacy: unable to read & $78.3(622)$ & $72.2(483)$ & $73.6(598)$ & $74.8(1703)$ & 0.015 \\
\hline Debt: currently in debt & $83.1(660)$ & $80.3(537)$ & $82.2(668)$ & $81.9(1865)$ & 0.360 \\
\hline Most sex work venue & & & & & 0.000 \\
\hline Brothel & $5.0(40)$ & $12.6(84)$ & $3.8(31)$ & $6.8(155)$ & \\
\hline Street & $11.1(88)$ & $17.6(118)$ & $4.7(38)$ & $10.7(244)$ & \\
\hline Lodge/hotel & $3.7(29)$ & $1.9(13)$ & $1.6(13)$ & $2.4(55)$ & \\
\hline Home & $22.0(175)$ & $25.1(168)$ & $19.5(159)$ & $22.1(502)$ & \\
\hline Highway & $21.7(172)$ & $12.4(83)$ & $5.9(48)$ & $13.3(303)$ & \\
\hline Agriculture or other venue & $22.4(178)$ & $10.0(67)$ & $24.5(199)$ & $19.5(444)$ & \\
\hline Multiple venues & $14.1(112)$ & $20.3(136)$ & $40.0(325)$ & $25.2(573)$ & \\
\hline Programme exposure & & & & & 0.000 \\
\hline No exposure & $57.0(453)$ & $30.9(207)$ & $33.2(270)$ & $40.8(930)$ & \\
\hline Receptive exposure only: & $23.6(187)$ & $22.1(148)$ & $18.3(149)$ & $21.3(484)$ & \\
\hline Aware of programme & (187) & (148) & $(148)$ & (483) & \\
\hline Received pamphlets & $(58)$ & (65) & (74) & (197) & \\
\hline Visited by programme staff & (92) & $(75)$ & (99) & $(266)$ & \\
\hline Active utilisation: & $19.4(154)$ & $46.9(314)$ & $48.5(394)$ & $37.9(862)$ & \\
\hline Visited drop-in canter (asked only in round 1) & $(125)$ & - & - & $(125)$ & \\
\hline Received medical care from programme clinic & $(95)$ & $(314)$ & $(394)$ & (803) & \\
\hline $\begin{array}{l}\text { Consistent condom use with non-clients (boyfriends, } \\
\text { lovers, husbands) in the past } 7 \text { days } \neq\end{array}$ & $30.9(111)$ & $39.9(122)$ & $44.4(186)$ & 38.7 (419) & \\
\hline \multicolumn{6}{|l|}{ Consistent condom use with clients in the past 7 days } \\
\hline All clients & $56.3(447)$ & $72.0(482)$ & $75.3(612)$ & $67.7(1541)$ & 0.000 \\
\hline Regular clients $\ddagger$ & $59.2(372)$ & 74.0 (382) & 77.4 (496) & $70.3(1250)$ & 0.000 \\
\hline Occasional clients $\ddagger$ & $64.5(461)$ & $76.9(463)$ & $80.1(564)$ & 73.6 (1488) & 0.000 \\
\hline
\end{tabular}


consistent condom use was $77 \%$ among FSWs who could read as compared with $65 \%$ among those who could not. With regard to venue, FSWs working in brothels, homes and highway settings had the highest proportions of consistent condom use. In addition, FSWs with higher numbers of clients in the past week were more likely to report using condoms consistently. Finally, programme exposure was highly associated with condom use: $55.7 \%$ of FSWs with no programme exposure reported consistent condom use with clients compared with $71.1 \%$ of those who had receptive exposure and $78.8 \%$ of those who actively utilised the intervention.

The patterns of association identified in bivariate analyses held up in multivariate analysis, as summarised in table 2: the first column presents the unadjusted percentages from bivariate analyses and the second column presents the adjusted ORs from the full multivariate model. Logistic regression results showed that age, age at start of sex work, literacy, venue and programme exposure were all significantly associated with consistent condom use with clients. Each 1-year increase in sex workers' age was associated with $2 \%$ lower odds of condom use. Women who entered sex work between ages 30 and 39 had 32\% lower odds of condom use as compared with women who entered before age 18 (OR 0.68, 95\% CI 0.49 to 0.96). Strikingly, women working in street-based venues had $61 \%$ lower odds of condom use as compared with women working in brothels (OR 0.59, $95 \% \mathrm{CI} 0.37$ to 0.95$)$. On the other hand, women living in conditions with more amenities present had greater odds of consistent condom use; an increase of only one amenity (electricity, running water, toilet or telephone) was associated with $25 \%$ greater odds of consistent condom use (OR 1.25, 95\% CI 1.14 to 1.36). Increased programme exposure was associated with increased likelihood of condom use: as compared with

Table 2 OR of the likelihood of consistent condom use with clients $(\mathrm{n}=2276)$

\begin{tabular}{|c|c|c|}
\hline & Percentage & Adjusted OR (95\% CI) \\
\hline Age & & $0.98^{* *}(0.97$ to 0.99$)$ \\
\hline \multicolumn{3}{|l|}{ Age at start of sex work } \\
\hline$<18$ years & 76.5 & Ref. \\
\hline $18-29$ & 68.5 & 0.81 (0.62 to 1.07 ) \\
\hline $30-39$ & 58.2 & $0.68^{*}(0.49$ to 0.96$)$ \\
\hline 40 years or older & 41.7 & $0.57(0.28$ to 1.13$)$ \\
\hline Literacy: unable to read & 64.6 & 1.20 (0.95 to 1.53$)$ \\
\hline Living conditions & & $1.25^{* * *}(1.14$ to 1.36$)$ \\
\hline \multicolumn{3}{|l|}{ Most sex work venue } \\
\hline Brothel & 76.8 & Ref. \\
\hline Street & 52.0 & $0.59 *(0.37$ to 0.95$)$ \\
\hline Lodge/hotel & 61.8 & 0.61 (0.31 to 1.22 ) \\
\hline Home & 76.5 & $1.22(0.77$ to 1.91$)$ \\
\hline Highway & 69.6 & $1.28(0.80$ to 2.06$)$ \\
\hline Agriculture or other venue & 63.5 & 0.88 (0.56 to 1.39 ) \\
\hline Multiple venues & 67.0 & 0.67 (0.43 to 1.03$)$ \\
\hline High frequency of sex trades & 71.3 & $1.12(0.90$ to 1.40$)$ \\
\hline \multicolumn{3}{|l|}{ Programme exposure } \\
\hline No exposure & 55.7 & Ref. \\
\hline Receptive exposure & 71.1 & $1.57^{* * *}$ (1.22 to 2.02$)$ \\
\hline Active utilisation & 78.8 & $2.03^{* * *}$ (1.60 to 2.57$)$ \\
\hline \multicolumn{3}{|l|}{ Survey round } \\
\hline Round 1 (2006) & 56.3 & Ref. \\
\hline Round 2 (2007) & 72.0 & $1.68^{* * *}$ (1.32 to 2.14$)$ \\
\hline Round $3(2009-2010)$ & 75.3 & $1.92 * * *(1.50$ to 2.46$)$ \\
\hline
\end{tabular}

Percentages are unadjusted. Adjusted ORs are from the regression model including all independent variables shown in the table.

${ }^{*} p<0.05,{ }^{* *} p<0.01$, ${ }^{* * *} p<0.001$.
FSWs with no programme exposure, FSWs with receptive exposure to the intervention had nearly two times the odds of consistent condom use with clients (OR 1.57, 95\% CI 1.22 to 2.02) and those who reported active utilisation had more than two times the odds (OR 2.03, 95\% CI 1.60 to 2.57). There was a main effect for data collection round, with greater likelihood of condom use among participants in both 2007 (OR 1.68, 95\% CI 1.32 to 2.14 ) and $2009-2010$ (OR $1.92,95 \%$ CI 1.50 to 2.46 ) compared with 2006.

As illustrated in figure 1, although the difference between rounds 1 and 2 was not statistically significant, consistent condom use with clients showed a trend of increasing over time (adjusted estimates from multivariate model of $56.3 \%$ in 2006 , $72.0 \%$ in 2007 and $75.3 \%$ in 2009-2010). Subsequent analysis including interactions between round and the other independent variables indicated no statistically significant interactions (results not shown). The association between programme exposure and consistent condom use with clients did not vary significantly over time, as indicated by the lack of significance of interaction terms for (programme exposure $\times$ round) ( $p$ values ranging from 0.22 to 0.58 ). This is also shown in figure 1 , which indicates that programme exposure remained an important predictor of consistent condom use with clients in each round of data collection. At each time point, highest proportions of consistent condom use were observed among FSWs with active utilisation of the intervention, followed by those with receptive exposure and finally those with no exposure.

\section{DISCUSSION}

We find that active utilisation of the CMI increased over time and consistent condom use with clients also increased. Programme exposure was significantly associated with consistent condom use and remained so over time. There was also some evidence of a dose-response: a stronger association between active intervention utilisation and consistent condom use than between receptive intervention exposure and consistent condom use. These findings suggest improvements in HIV risk reduction behaviours over time in FSWs in Rajahmundry, the target population of a long-standing CMI. The level of programme exposure we discovered is consistent with Steen et $a l^{26}$ who found that following STI interventions implemented as part of Avahan (principally in southern India), 70\% of FSWs in the Avahan coverage area had received peer outreach and $41 \%$ had visited an STI clinic. It also builds upon earlier work from Project Parivartan, ${ }^{23}$ which examined programme exposure and its correlates in the first and second rounds of data collection.

Our results suggest that the CMI may be contributing to population-level HIV risk reduction among FSWs. Although

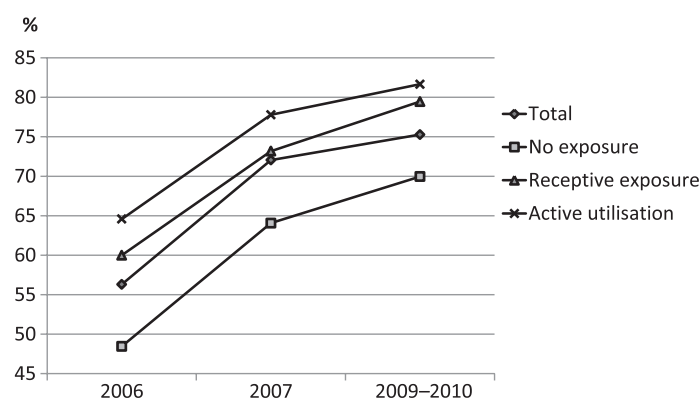

Figure 1 Consistent condom use with clients by programme exposure and survey round (adjusted percentages estimated from multivariate model). 
causality cannot be inferred from our study, the findings are consistent with a sustained effect of the intervention. To the extent that the trend in condom use increased over time independently of involvement in the intervention, it may imply that the effect of the intervention is being felt even by those FSWs who have not been directly exposed to it. However, achieving and maintaining a high level of active utilisation of the intervention is not only possible but also likely with CMIs because intervention strategies are collectively chosen by members of the target population and the intervention thus adapts to the population needs over time.

The findings of this study should be interpreted in light of several study limitations. First, the data analysed in this study were cross-sectional, restricting our ability to ascertain causality. Because the cross-sectional surveys were anonymous, we were unable to determine which respondents had participated in more than one round of data collection. Future CMIs should consider longitudinal data collection, when feasible, to confirm the observed associations and establish temporal order. This analysis also focused exclusively on adult FSWs over age 18; no conclusions can be drawn with regard to condom use or the association of programme exposure with condom use for younger sex workers. Presumably, some of the collective mobilisation activities and changes in norms and policies would benefit younger sex workers as well. In addition, we face potential limitations related to self-reported data. The accuracy and reliability of FSW reports of either condom use with clients or exposure to the intervention in this context is unknown. Other studies have shown a tendency to under-report sensitive issues or socially undesirable behaviours ${ }^{27}$; such under-reporting would tend to decrease power to detect an association between programme exposure and consistent condom use. Although there is also the possibility that social desirability might increase with intervention exposure, resulting in overestimation of the effect on condom use, our findings are congruent with other similar HIV interventions in this context that have used biological markers for STDs and HIV. Current HIV prevention interventions have been associated with reduced HIV risk behaviours as well as declining HIV risk among FSWs in varied south Indian settings. ${ }^{6} 7$ Reza-Paul and colleagues ${ }^{7}$ demonstrated both increases in condom use (from $65 \%$ to $90 \%$ with occasional clients and from $53 \%$ to $66 \%$ with repeat clients) and declines in STI prevalence (syphilis $25 \%$ to $12 \%$, chlamydia $11 \%$ to $5 \%$ and gonorrhoea $5 \%$ to $2 \%$ ) over a 2.5 -year period of a comprehensive FSW community-led HIV intervention. Also in Karnataka state, a recent study by Ramesh and colleagues ${ }^{6}$ showed similar declines associated with HIV interventions among FSWs (condom use increased from $83 \%$ to $88 \%$ with occasional clients and from $66 \%$ to $84 \%$ with repeat clients; syphilis declined from $6 \%$ to $3 \%$ and chlamydia and/or gonorrhoea from $9 \%$ to $7 \%$ ). Furthermore, current country estimates suggest that the epidemic is declining among antenatal clinic attendees as well as among populations at greatest risk for HIV, including FSWs. ${ }^{4}$ Such declines have been attributed to current HIV prevention programmes. ${ }^{28}$ The results of our study and others thus suggest positive promising movement towards reducing HIV in high-risk groups in India. Finally, the study employed RDS to recruit respondents. RDS can achieve an unbiased and representative sample only when certain statistical requirements are met. ${ }^{29} 30$ Although we cannot be certain, we achieved a sample truly representative of the underlying population, numerous prior studies have established RDS as an effective method to sample hard-to-reach and hidden populations such as FSW. ${ }^{31-34}$

\section{What is already known on this subject}

CMls have shown promise to address HIV risk among FSWs.

- Such interventions organise FSWs for collective action and challenge the structures of power that underlie their HIV risk.

- Evaluating the impact of CMls is challenging because the intended change occurs at structural in addition to individual levels.

- In areas with long-standing and sustained CMls, it is unknown whether direct exposure to intervention components remains important over time for improvements in HIV risk reduction behaviour.

\section{What this study adds}

- In this study, we analyse survey data from three repeated cross sections of FSWs in Rajahmundry, India.

- We find that active utilisation of the intervention increased over time, and consistent condom use with clients also increased.

- Programme exposure was significantly associated with consistent condom use and remained so over time.

- Our findings suggest that the CMI may be contributing to population-level HIV risk reduction among FSWs.

- Sustaining a high level of programme exposure is not only possible but also likely with CMls because intervention strategies are collectively chosen by members of the target population and the intervention thus adapts to the target population's needs over time.

These limitations notwithstanding, the current study findings indicate improvements in HIV risk reduction behaviour among FSWs throughout the duration of a CMI. The significant relation between programme exposure and consistent condom use throughout the three waves of data collection suggests that this CMI had substantial reach in the FSW population and may likely be contributing to population-level HIV risk reduction among FSWs. Our findings build on the increasing number of studies in India, as well as elsewhere, showing the importance of structural interventions for addressing HIV risk among a broad range of populations most vulnerable to HIV.

Acknowledgements The authors wish to thank all the women who participated in the cross-sectional surveys, the Rajahmundry NGO CARE-Saksham, and Project Parivartan team members in India and the USA

Contributors JTE conceptualised data analysis, analysed the data and drafted and revised the paper. She is the guarantor. KMB designed data collection tools, oversaw all Project Parivartan data collection, conceptualised data analysis and drafted and revised the paper. MB and ER designed data collection tools, oversaw Project

Parivartan data collection and drafted and revised the paper. RB provided guidance for statistical analysis and drafted and revised the paper.

Funding This study was supported by Bill and Melinda Gates Foundation through the Avahan India AIDS Initiative. The views expressed herein are those of the authors and do not necessarily reflect the official policy or position of the Bill \& Melinda Gates Foundation and Avahan.

\section{Competing interests None.}

Ethics approval Ethics approval was provided by ethical review boards at American University, Duke University, Yale University and YRG-Care (Chennai, India).

Provenance and peer review Commissioned; externally peer reviewed. 
Data sharing statement Data from this study are available for research purposes upon written request to the study PI (KMB).

\section{REFERENCES}

1. UNAIDS. Global Report: UNAIDS Report on the Global AIDS Epidemic 2010. Geneva: Joint United Nations Programme on HIV/AIDS (UNAIDS), 2010.

2. Ramesh BM, Moses S, Washington R, et al. Determinants of HIV prevalence among female sex workers in four south Indian states: analysis of cross-sectional surveys in twenty-three districts. AIDS 2008;22(Suppl 5):S35-44.

3. Jha P. Documenting the Geographic Variation in HIV-1, Its Determinants and Intervention Coverage in 115 Districts in Southern India. Toronto: Centre for Global Health Research, St. Michael's Hospital, University of Toronto, 2006

4. National AIDS Control Organization (NACO), , Government of India. Annual Report 2009-2010. Ministry of Health and Family Welfare, Department of AIDS Control, 2010. http://www.nacoonline.org/upload/AR\%202009-10/NACO_AR_ English\%20corrected.pdf (accessed 1 Dec 2011).

5. APSACS. Eighth Round of National Annual Sentinel Surveillance for HIV, Andhra Pradesh. Hyderabad: Andhra Pradesh AIDS Control Society (ASACS), 2004.

6. Ramesh BM, Beattie TS, Shajv l, et al. Changes in risk behaviours and prevalence of sexually transmitted infections following HIV prevention interventions among female sex workers in five districts in Karnataka state, south India. Sex Transm Infect 2010:86(Suppl 1):S91-100

7. Reza-Paul S, Beattie T, Syed HU, et al. Declines in risk behaviour and sexually transmitted infection prevalence following a community-led HIV preventive intervention among female sex workers in Mysore, India. AIDS 2008:22:91-100.

8. Chattopadhyay A, McKaig RG. Social development of commercial sex workers in India: an essential step in HIV/AIDS prevention. AIDS Patient Care STDs 2004;18:159-68.

9. Herek GM. AIDS and stigma. Am Behav Sci 1999;42:1102-12.

10. Parker R, Aggleton P. HIV and AIDS-related stigma and discrimination: a conceptual framework and implications for action. Soc Sci Med 2003:57:13-24.

11. Valdiserri R. AIDS stigma: an impediment to public health. Am J Public Health 2002:92:341-2

12. Parker R. The global HIV/AIDS pandemic, structural inequalities, and the politics of international health. Am J Public Health 2002;92:343-6.

13. Blankenship KM, Friedman SR, Dworkin S, et al. Structural interventions: concepts, challenges and opportunities for research. J Urban Health 2006:83:59-72.

14. Shamanesh M, Pate V, Mabey D, et al. Effectiveness of interventions for the prevention of HIV and other sexually transmitted infections in female sex workers in resource poor settings: a systematic review. Trop Med Int Health 2008;13:659-79.

15. Halli SS, Ramesh BM, O'Neill J, et al. The role of collectives in STI and HIV/AIDS prevention among female sex workers in Karnataka, India. AIDS Care 2006;18:739-49.

16. Blankenship KM, West BS, Kershaw TS, et al. Power, community mobilization, and condom use practices among female sex workers in Andhra Pradesh, India. AIDS 2008;22(Suppl 5):S109-16
17. Basu I, Jana S, Rotheram-Borus MJ, et al. HIV prevention among sex workers in India. J Acquir Immune Defic Syndr 2004;36:845-52.

18. Swendeman D, Basu I, Das S, et al. Empowering sex workers in India to reduce vulnerability to HIV and sexually transmitted diseases. Soc Sci Med 2009;69:1157-66.

19. Evans C, Jana S, Lambert H. What makes a structural intervention? Reducing vulnerability to HIV in community settings, with particular reference to sex work. Glob Public Health 2010;5:449-61.

20. Auerbach JD, Parkhurst JO, Caceres CF. Addressing social drivers of HIV/AIDS for the long-term response: conceptual and methodological considerations. Glob Public Health 2011;6(Suppl 3):S293-309.

21. Jana S, Basu I, Rotheram-Borus MJ, et al. The Sonagachi project: a sustainable community intervention program. AIDS Educ Prev 2004;16:405-14.

22. Pardasani MP. HIV prevention and sex workers: an international lesson in empowerment. Int J Soc Welfare 2005:14:116-26.

23. Blankenship KM, Burroway R, Reed E. Factors associated with awareness and utilisation of a community mobilisation intervention for female sex workers in Andhra Pradesh, India. Sex Transm Infect 2010;86(Suppl 1):i69-75.

24. Heckathorn DD. Respondent-driven sampling II: deriving valid population estimates from chain-referral samples of hidden populations. Soc problems 2002:49:11-34.

25. Stata Statistical Software: Release 10.1 [computer Program]. College Station, TX: StataCorp LP, 2009.

26. Steen R, Mogasale V, Wi T, et al. Pursuing scale and quality in STI interventions with sex workers: initial results from Avahan India AIDS Initiative. Sex Transm Infect 2006:82:381-5.

27. Hanck SE, Blankenship KM, Irwin KS, et al. Assessment of self-reported sexual behavior and condom use among female sex workers in India using a polling box approach: a preliminary report. Sex Transm Dis 2008;35:489

28. UNAIDS/WHO. Country Situation: India 2009. Geneva: UNAIDS/WHO, 2010. http:// www.unaids.org/ctrysa/asiind en.pdf (accessed 1 Dec 2011).

29. Heimer R. Critical issues and further questions about respondent-driven sampling: comment on Ramirez-Valles, et al. (2005). AIDS Behav 2005;9:403-8.

30. Salganik MJ, Heckathorn DD. Making unbiased estimates about hidden populations using respondent-driven sampling. Sociol Methodol 2004;34:193-240.

31. Abdul-Quader AS, Heckathorn DD, McKnight C, et al. Effectiveness of respondentdriven sampling for recruiting drug users in New York City: findings from a pilot study. $J$ Urban Health 2006;83:459-76.

32. Johnston LG, Sabin K, Hien MT, et al. Assessment of respondent driven sampling for recruiting female sex workers in two Vietnamese cities: reaching the unseen sex worker. J Urban Health 2006;83:116-28.

33. Salganik MJ, Heckathorn DD. Sampling and estimation in hidden populations using respondent-driven sampling. Sociol Methodol 2004;34:193-239.

34. Simic M, Johnston LG, Platt L, et al. Exploring barriers to 'respondent driven sampling' in sex worker and drug-injecting sex worker populations in Eastern Europe. J Urban Health 2006;83:i6-15. 